\title{
A Method for Investigation of the Galactic Magnetic Field using Ultra-High Energy Cosmic Rays
}

\section{Martin Erdmann*, Gero Müller, Martin Urban}

Phys. Institute 3A, RWTH Aachen University, D-52056 Aachen, Germany

E-mail: erdmann@physik.rwth-aachen.de

\begin{abstract}
We present a method to investigate deflections of ultra-high energy cosmic rays in the galactic magnetic field. A key element of our analysis are simulations of expected arrival directions of the cosmic rays after traversing the field. The method enables tests of cosmic ray sources, composition, and magnetic field deflections. It is suited for astrophysical scenarios which have on top of a dominating isotropic background a few cosmic rays each originating from a different source.
\end{abstract}

The European Physical Society Conference on High Energy Physics 22-29 July 2015

Vienna, Austria

* Speaker. 


\section{Introduction}

Ultra-high energy cosmic rays are presumed to be deflected in extragalactic and galactic magnetic fields prior to their observation. For these deflections the directional dependence of the field pattern and the directional variations in the magnitude of the fields are most relevant. In another view, cosmic rays provide information on magnetic fields they traversed. Once these fields are known they could serve as spectrometer magnets to identify protons, or enable measurements of cosmic ray composition.

Regarding the description of the galactic magnetic field substantial progress has been made in recent years. Based on Faraday rotation and synchrotron emission measurements, parameterizations have been developed that enable detailed predictions for deflection of cosmic particles as a function of their energy and charge $[1,2,3,4,5]$. It needs to be investigated whether these parameterizations adequately represent the directionality and strength of the magnetic field as realized in our galaxy, and whether cosmic particles are deflected accordingly. To perform a simple test one would need an extragalactic source; however, since a source has not been identified to date, the magnetic field needs be tested simultaneously with a hypothesis of preferred directions of cosmic rays outside of our galaxy.

Our analysis strategy is to investigate a combined model with three assumptions (Fig. 1a):

1. The first assumption is that coincident directions of cosmic rays with source candidates exist outside of our galaxy, before the cosmic rays are deflected in the galactic magnetic field.

2. The second aspect of the model is that the above parameterizations of the galactic magnetic field reflect the field characteristics and magnitude as realized in our galaxy.

3. The third assumption is that protons exist in the considered cosmic ray data sample, and that the magnetic field separates protons and heavy nuclei.

Tests of this model can be performed for different combinations of the three assumptions (Fig. 1a). For example, for testing the validity of the hypothesis of coincident directions of cosmic ray protons (3) and the selected source candidates (1) we assume the field parameterization (2) to be valid and perform a test with isotropically distributed source directions. To test the directional characteristics of the field (assumption 2) we perform a test with reversed magnetic field directions.

By performing such tests we obtain combined information on the validity of the magnetic field corrections, on the proton contribution in the cosmic ray data, and on the validity of the hypothesis of coincident cosmic ray and source directions. Note that owing to the forward simulation technique we can test the validity of a model without imposing potentially unphysical conditions on measured cosmic rays which is a typical drawback to be solved in cosmic ray backtracking methods. Also note that each cosmic ray and source is treated separately such that the method is suited for astrophysical situations where each observed cosmic ray originates from a different source.

\section{Simulated astrophysical scenario}

We assume that a subset of active galactic nuclei (AGN) [6] can be considered as sources of cosmic rays. We used high energy neutrinos [7] with energies $E>40 \mathrm{TeV}$ and no atmospheric 
a)

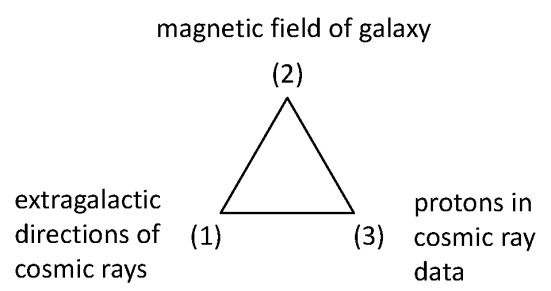

c)

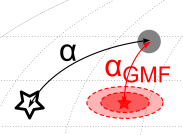

b)

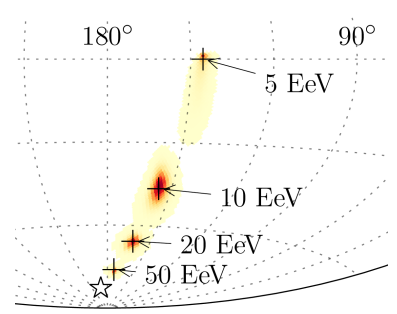

d)

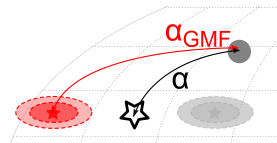

Figure 1: a) Visualization of the 3 model assumptions on the (1) directions of cosmic rays outside our galaxy, (2) parameterization of the galactic magnetic field, and (3) existence of protons in the cosmic ray sample. b) Visualization of the magnetic field correction. The star symbol denotes the cosmic ray direction outside the galaxy, the cross symbols show the energy dependency of the expected arrival directions of protons, and the color code gives relative probability distributions. c) Angular distances of a cosmic ray (full symbol) to an AGN direction (star symbol) and to the expected arrival direction (colored area). d) Visualization of the expected arrival direction of reversed magnetic field directions.

assignment as experimental navigators and selected their nearest AGNs with distances $z<0.018$ [8]. This selection provided 22 AGN sources within the GZK horizon [9].

With the new CRPropa program version 3 [10] we generated $10^{7}$ cosmic nuclei with charges between $Z=1, \ldots, 26$ at each of these AGN sources. Upon arrival at a $0.5 \mathrm{Mpc}$ observer sphere around our galaxy we use galactic magnetic field lenses to project the arriving cosmic rays onto Earth. The lenses consist of matrices based on the HEALPix format [11] where we divide the sphere into 49,152 equally sized pixels of approximately $1 \mathrm{deg}$. The matrices contain the probability of a cosmic ray entering the galaxy with energy $E$ at direction $i$ to be observed in direction $j$. The technical details of the lenses and their production are outlined in [12]. The lenses used in this contribution have been calculated with the CRPropa program and the JF12 parameterization of the galactic magnetic field [2].

In order to obtain a simulated cosmic ray data set of $N=231$ ultra-high energy cosmic rays with energies above $E=52 \mathrm{EeV}$, which corresponds to the currently published statistics of the Pierre Auger Observatory [13], we combine 90\% isotropic cosmic rays with $10 \%$ cosmic rays from the CRPropa simulation. The total sample contains $7 \%$ signal protons which are related to the AGN sources. The simulated cosmic rays follow the geometrical acceptance of the observatory [14], and an energy distribution corresponding to the measured energy spectrum [15].

\section{Expected arrival directions of protons}

We perform the following analysis without information on the simulated cosmic ray particle type and history. In the first step we calculate the expected arrival directions of the cosmic rays assuming they are protons. In Fig. $1 \mathrm{~b}$ the procedure is visualized exemplarily for protons with $E=5,10,20,50 \mathrm{EeV}$ arriving from a source which is represented by the star symbol. 
We start outside the galaxy with the directions of the selected AGNs mentioned above. To take into account effects of extragalactic magnetic fields we apply a Fisher probability distribution centered at each AGN direction with a concentration parameter $\kappa$ depending on the AGN distance and the cosmic ray energy. For an AGN at distance $D=10 \mathrm{Mpc}$ and a proton with $E=52 \mathrm{EeV}$ the angular spread amounts to $\sigma=1 / \sqrt{\kappa}=6 \mathrm{deg}$. This probability distribution is then projected onto the Earth using the magnetic lensing techniques mentioned above. The relative arrival probabilities at Earth are also shown in the figure with the color code. To obtain a direction that will yield the largest arrival probability, we calculate the radius $r_{50}$ containing $50 \%$ of the arrival probabilities. The center of the pixel with the smallest radius $r_{50}$ is then used as the expected arrival direction (cross symbols).

The direction of the lowest proton energy $E=5 \mathrm{EeV}$ also corresponds to the deflection of an ionized Neon nucleus with $E=50 \mathrm{EeV}$ energy and positive charge $Z=10$. Therefore, if coincident directions of protons in the cosmic ray sample and AGNs exist, the protons can to some extent be identified by small angular distances to their expected arrival directions.

\section{Angular distances and clustering strength}

In Fig. 1c we visualize the angle $\alpha$ which denotes the angular distance between the measured arrival directions of a cosmic ray and its nearest AGN. The angle $\alpha_{\mathrm{GMF}}$ denotes the angular distance between the measured cosmic ray and the expected arrival direction of a proton with an AGNcoincident direction outside the galaxy.

We measure the improvement in the angular distance by the difference in the two angles. In Fig. 2a, the distribution of $\alpha-\alpha_{\mathrm{GMF}}$ is shown as a function of the minimum of the two angular distances. At positive values the cosmic rays come closer to the expected arrival directions, while at negative values the cosmic rays are nearer to the AGNs without field corrections. The cosmic rays at small angular distances reveal the proton contribution in the cosmic ray event sample (assumption 3 on composition in section 1).

We quantify the change in the angular distances by the asymmetry

$$
A \equiv 2 \frac{N\left(\alpha>\alpha_{\mathrm{GMF}}\right)-N\left(\alpha<\alpha_{\mathrm{GMF}}\right)}{N\left(\alpha>\alpha_{\mathrm{GMF}}\right)+N\left(\alpha<\alpha_{\mathrm{GMF}}\right)}
$$

which can take on any value from -2 to 2 . Taking, e.g., a maximum angle of 5 deg the angular asymmetry in the data is found to be $A=0.96$.

We also investigate clustering of cosmic rays with AGN directions. In Fig. $2 b$ we present the frequencies of the cluster sizes $m$ where we count associations of cosmic rays with AGNs within 5 deg angular distance. This yields configurations containing singlet, doublet, ... clusters $(m=$ $1,2, \ldots, 6)$. The triangular symbols represent the data when including magnetic field corrections, and the histogram without them.

To quantify the observed clustering strength we use the multinomial probability distribution:

$$
P\left(n_{1}, \ldots, n_{22} ; N-N_{h i t}\right)=\frac{N !}{n_{1} ! \ldots n_{22} !\left(N-N_{h i t}\right) !} p_{1}^{n_{1}} \ldots p_{22}^{n_{22}}\left(1-p_{\text {iso }}\right)^{N-N_{\text {hit }}}
$$

The value $P$ describes the expected level of trivial clustering between the $N=231$ cosmic rays, and the 22 AGNs where the latter are distinguished by identifiers. AGN $i$ has an average hit probability 
a)

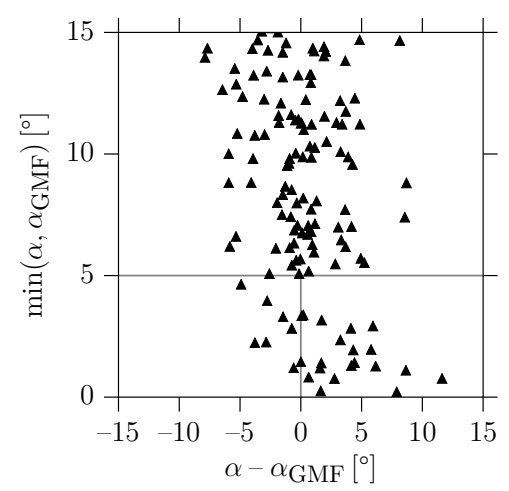

c)

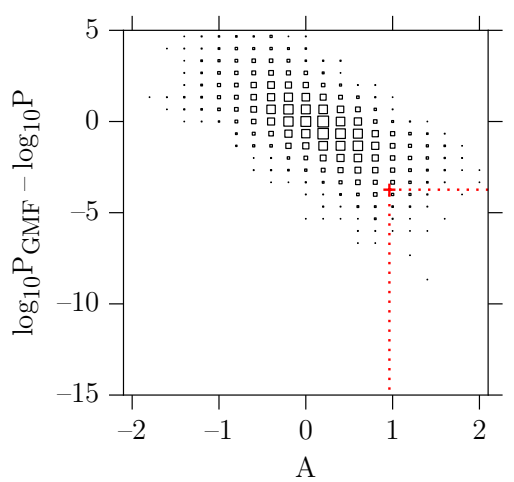

b)

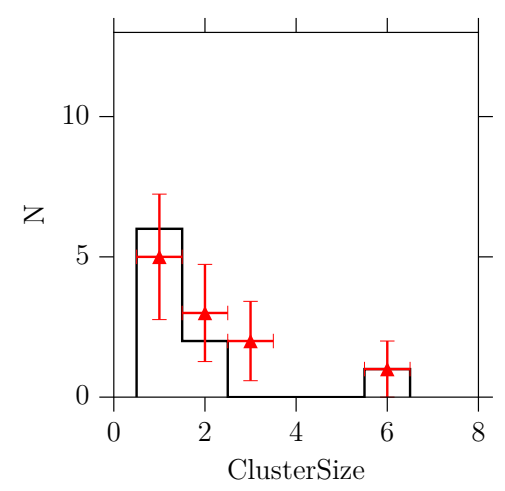

d)

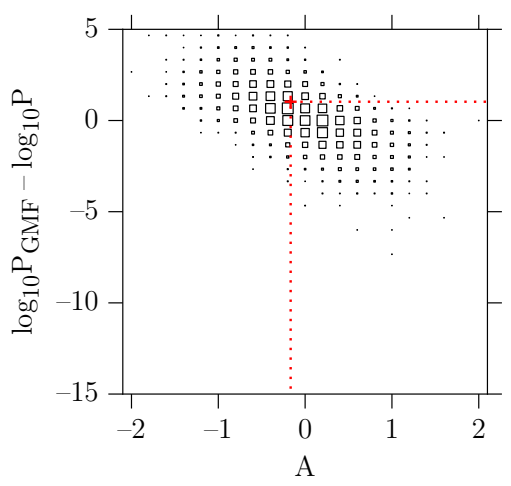

Figure 2: a) Change in the angular distance before and after applying the magnetic field corrections. The vertical line separates reduced $(>0)$ and enlarged $(<0)$ angular distances. The vertical axis shows the smallest angular distance, whereas the horizontal line emphasizes the region below $5 \mathrm{deg}$. b) Frequencies of the cluster sizes $m$ for the maximum angular distance $5 \mathrm{deg}$. c) Improvement in the clustering strength versus the angular asymmetry for the nominal data analysis (red cross symbol) and simulated analyses using isotropic sources (box symbols). d) Data analysis with reverse field directions (red cross symbol), the box symbols show analyses using isotropic cosmic rays and random field directions.

of $p_{i}$, and was correlated with $n_{i}$ cosmic rays. The total number of cosmic rays associated with one of the AGNs is $N_{h i t}=\sum_{i} n_{i}$. The remaining $N-N_{h i t}$ cosmic rays without AGN correlations had a no-hit probability of $\left(1-p_{i s o}\right)$. Summing the hit probabilities for the AGNs at their nominal arrival directions for angular distances below 5 deg gives $p_{i s o}=5.3 \%$. This includes the geometric acceptance of the Pierre Auger Observatory [14]. When including the magnetic field corrections a slight energy dependence is observed where the probability increases on average to $p_{\text {iso }}=5.7 \%$.

When applying the magnetic field corrections to the data, the logarithm of the multinomial probability (4.2) amounts to $\log _{10}\left(P_{\mathrm{GMF}}\right)=-15.3$. Without the field corrections the level of clustering is smaller and results in $\log _{10}(P)=-11.5$. The change in the clustering strength thus amounts to $\log _{10}\left(P_{\mathrm{GMF}}\right)-\log _{10}(P)=-3.8$. In Fig. $2 \mathrm{c}$ this value is shown together with the asymmetry value (4.1) as the red cross symbol.

\section{Discussion}

In order to test the analysis method we exploit the assumptions of our combined model represented by the triangle in Fig. 1a with respect to isotropic scenarios. First we use the nominal cosmic rays and the correct galactic field as explained in section 2, however, we use 22 isotropic 
AGN directions instead of the true AGN directions. In Fig. 2c we compare the result of the nominal analysis (red cross) with 10,000 simulations of analyses using the wrong sources (box symbols). Of these analyses only $0.47 \%$ have equal values or improvements compared to the values obtained in the nominal data analysis (lower right area of Fig. 2c). Therefore, the correct sources can be identified even in a cosmic ray data sample which is dominated by isotropic background.

In a second test we use the nominal cosmic rays and the correct AGN sources, however, we use lenses produced for correcting deflections of antiprotons in the galactic magnetic field. These lenses reverse the galactic magnetic field for protons (Fig. 1d). Applying the reversed field in our data analysis instead of the correct field orientation, the angular asymmetry and the change in the clustering strength both diminish (red cross in Fig. 2d). To validate that these values cannot be distinguished from isotropic scenarios we simulated 10.000 analyses with the correct AGN directions, however with isotropic cosmic rays and random directions of the galactic field (box symbols). Thus, the second assumption in section 2 on the validity of the field parameterizations can be exploited in comparison with analyses using random field directions and field reversal.

We thus expect that by applying our method to data, cosmic ray deflections in the galactic field can be verified experimentally. Once this has been established, our method can further be used to gather information on details of the galactic magnetic field and cosmic ray sources.

\section{Acknowledgments}

We are grateful for financial support to the Ministerium für Innovation, Wissenschaft und Forschung des Landes Nordrhein-Westfalen and to the Bundesministerium für Bildung und Forschung (BMBF), and for helpful comments on the manuscript to R. Clay.

\section{References}

[1] M. S. Pshirkov, P. G. Tinyakov, P. P. Kronberg, K. J. Newton-McGee, ApJ 738 (2011) 192

[2] R. Jansson, G. R. Farrar, ApJ 757 (2012) 14

[3] R. Jansson, G. R. Farrar, ApJ 761 (2012) L11

[4] M. S. Pshirkov, P. G. Tinyakov, F. R. Urban, Mon. Not. Roy. Astron. Soc. 436 (2013) 2326

[5] M. C. Beck, A. M. Beck, R. Beck, K. Dolag, A. W. Strong, P. Nielaba, 2014, arXiv:1409.5120

[6] M.-P. Véron-Cetty, P. Véron, $A \& A$ 455, 2, 2006, 773;

[7] The IceCube Collaboration, M. G. Aartsen et al., Phys. Rev. Lett. 113 (2014) 101101

[8] The Pierre Auger Collaboration, J. Abraham et al, (9 November 2007) Science 318 (5852) 938

[9] K. Greisen, PRL 16 (1966) 748; G. T. Zatsepin, V. A. Kuz'min, JETP Letters 4 (1966) 78

[10] R. A. Batista et al., 2014, arXiv:1410.5323

[11] K. Gorski, E. Hivon, A. Banday, B. Wandelt, F. Hansen, et al., ApJ 622 (2005) 759

[12] H.-P. Bretz, M. Erdmann, P. Schiffer, D. Walz, T. Winchen, Astropart. Phys. C 54 (2014) 110

[13] The Pierre Auger Collaboration, A. Aab et al., ApJ 804 (2015) 15

[14] P. Sommers, Astropart. Phys. 14 (2001) 271

[15] The Pierre Auger Collaboration, J. Abraham et al., Phys. Letters B685 (2010) 239 